\title{
Evaluación comparativa de los museos de sitio de Pachacamac, Puruchuco y Huaca Pucllana, según la percepción de los estudiantes de turismo en Lima Metropolitana
}

\author{
Comparative evaluation of the Pachacamac, Puruchuco \\ and Huaca Pucllana site museums, according to the \\ perception of tourism students in Metropolitan Lima
}

(iD) Florisa García Chumioque ${ }^{\varpi_{y}}$ (iD) Pedro Pizarro Ramos

Universidad Le Cordon Bleu. Lima, Perú

\begin{tabular}{llll}
\hline Recibido: $17 / 04 / 2018$ & Revisado: 27/04/2019 & Aceptado: 16/05/2019 & Publicado: 30/06/2019
\end{tabular}

\section{RESUMEN}

En esta investigación se realizó una evaluación comparativa respecto a los museos en mención según la percepción de los estudiantes de turismo en Lima Metropolitana.

El objetivo de la presente investigación es "Elaborar una evaluación comparativa de los museos de sitio Pachacamac, Puruchuco y Huaca Pucllana según la percepción del visitante residente en la ciudad de Lima". Para realizar este trabajo de investigación, se realizaron varias sesiones de debates entre los investigadores en donde se analizó la realidad situacional de los museos de sitio en la ciudad de Lima, concluimos que lo más oportuno sería desarrollar una evaluación comparativa de los principales museos de sitio que tiene Lima Metropolitana.

Una vez elegido el tema de investigación se realizó la búsqueda y recopilación de información pertinente a través del internet, repositorios, revistas especializadas, artículos, entre otras fuentes. Como parte de los resultados, la muestra estuvo conformada por participantes del sexo masculino y femenino. También estuvo conformada por grupos etarios los mismos que oscilan entre los "0 - 20", "21 - 40", "41 - 60" años. La muestra encuestada pertenece a la universidad privada $A$, universidad privada $B$, universidad nacional $A$, instituto $A$, instituto $B$, instituto $C$.

Por otro lado, el museo que ha venido mostrando mejoras respecto al paso de los años, es el museo de sitio de Pachacamac. Respecto a la evaluación del "diseño de la exhibición permanente", los museos de sitio Pachacamac y Puruchuco obtuvieron la calificación de "muy satisfecho" y el museo de sitio Huaca Pucllana obtuvo la calificación de "satisfecho".

Se concluye que los museos tienen una importancia vital en la formación de los futuros profesionales del sector turismo, garantizando así el sentido de pertenencia por parte de todos los peruanos y permitiendo elaborar una malla curricular acorde al contexto sociocultural peruano dentro de la globalización.

Palabras clave: Museo de sitio, Pachacamac, Puruchuco, Pucllana. 


\section{ABSTRACT}

In this research, a comparative evaluation was carried out regarding the mentioned museums according to the perception of tourism students in Metropolitan Lima.

The objective of the present investigation is "To prepare a comparative evaluation of the museums of Pachacamac, Puruchuco and Huaca Pucllana sites according to the perception of the visitor resident in the city of Lima". To carry out this research work, several discussion sessions were held among the researchers where the situational reality of the Site Museums in the city of Lima was analyzed, we concluded that the most appropriate thing would be to develop a comparative evaluation of the main Site museums that has Metropolitan Lima.

Once the research topic was chosen, the search and compilation of pertinent information was carried out through the internet, repositories, specialized magazines, articles, among other sources.

As part of the results, the sample consisted of male and female participants. It was also made up of age groups that range between " 0 - 20", "21 - 40", "41 - 60" years of age. The sample surveyed belong to the University Private A, University Private B, the National University A, the Institute A, the Institute B, the Institute $C$. On the other hand the museum that has been showing improvements over the years, is the Pachacamac Site Museum. Regarding the evaluation of the "Design of the Permanent Exhibition", the Pachacamac and Puruchuco Site Museums obtained the (Very Satisfied) qualification and the Huaca Pucllana Site Museum obtained the (Satisfied) qualification.

It is concluded that museums have a vital importance in the training of future professionals in the tourism sector, thus guaranteeing the sense of belonging on the part of all Peruvians and allowing the elaboration of a curricular mesh according to the Peruvian socio-cultural context within globalization.

Keywords: Museum of site, Pachacamac, Puruchuco, Pucllana.

\section{INTRODUCCIÓN}

El museo es una institución permanente, sin fines de lucro, al servicio de la sociedad y de su desarrollo, abierta al público y que efectúa investigaciones sobre testimonios materiales de la humanidad y de su medio ambiente, adquiridos, conservados, comunicados y, sobre todo, expuestos para fines de estudio, de educación y deleite.

El museo de sitio de Pachacamac, se sitúa en el distrito de Pachacamac y está ubicado al sur de la ciudad de Lima. Etimológicamente el nombre Pachacamac es un término de origen quechua, según el Inca Garcilaso de la Vega, el nombre es compuesto por: Pacha, significa mundo; Camac, que significa animar, así, Pachacamac significa el Dios que anima y vivifica el mundo.
Según el cronista Pedro Cieza de León, escribe lo siguiente: "El nombre de este demonio quería decir hacedor del mundo. Porque CAMAC quiere decir "hacedor" y PACHA "mundo" (Gobierno Municipal Pachacamac).

El valor histórico de Pachacámac recae en el santuario arqueológico del mismo nombre. Se trata de un conjunto arquitectónico en el que se evidencia el paso de distintas culturas, desde la cultura Lima, en el año 200 d.C. hasta la expansión incaica por los años 1470, durante el gobierno de Inca Yupanqui, y posteriormente la invasión hispana (Gobierno Municipal - Pachacamac).

Su importancia radica en ser uno de los principales santuarios de la costa peruana cuya divinidad aparece en los re-

Rev. Investigaciones ULCB. Ene - jun. 6(1), 2019; ISSN: 2409 - 1537; 53 - 62. 
latos coloniales como "dios costeño soberano en todos los yungas durante el último milenio".

Sus características en arquitectura y paisaje circundante le otorgan una belleza excepcional. A la llegada de los españoles, constituía el santuario más importante de la costa, espacio sagrado habitado por sacerdotes dedicados al culto del dios Pachacámac, al cual acudían peregrinos de distintas partes para pedir consejo y augurios.

Los incas establecieron en el lugar una importante capital provincial, donde destacaban el imponente templo del Sol y el Acllawasi (Ministerio de Cultura, 2010).

El museo de sitio de Puruchuco proviene del vocablo de origen quechua, según Arturo Jiménez Borja, fundador del museo de sitio de Puruchuco, significa casco emplumado, sombrero de plumas o algo semejante.

En 1988, Jiménez da referencias de otros nombres con los que se conocía al sitio arqueológico, "unas veces se le llama Pocorucha, Pocurucha, Pocorucho y Pocurcho" en síntesis el autor concluye que es probable que Puruchuco bien pudo ser el nombre de alguna persona como "el señor del sombrero de plumas".

El sitio arqueológico de Puruchuco data del Horizonte Tardío (entre 1438 y 1532) y fue la residencia de un líder local o curaca.

Como centro de distribución tuvo funciones de residencia y administrativas. Se ubica sobre las faldas del cerro Mayorazgo, en el antiguo fundo Vista Alegre por entonces propiedad del señor Alberto Isola, y ubicado a escasos 800 metros de la carretera central. En la actualidad esta zona pertenece a la urbanización Los Portales de Puruchuco, distrito de Ate (Amoros, 2017).

El museo de sitio Huaca Pucllana fue inaugurado en 1984, tuvo su primera intervención en 1967 por la doctora Isabel Flores por invitación del alcalde de Miraflores, Juan José Vega, quien fuera elegido alcalde en el año 1967 y al poco tiempo renunciaría.

En 1981, con el compromiso del Instituto Nacional de Cultura y la Municipalidad de Miraflores en el "Forum Taller Miraflores al 2000" se inició el proyecto de investigación, conservación y puesta en valor de la Huaca Pucllana, en el cual se reubicó a los invasores del sitio arqueológico.

Según (Miraflores, 2018) el 6 de febrero de 1987 Huaca Pucllana fue declarada zona arqueológica intangible y en 1989 se reconoció el museo como entidad cultural integrante del sistema nacional de museos y doce años después, la Huaca PucIlana es declarada Patrimonio Cultural de la Nación.

Los museos de sitio, según señala Chirinos (2013), las definiciones de Moolman respecto al término museo de sitio se utilizó por primera vez en la década de los años 50 en Francia como museé de site para referirse al trailside museum de Estados Unidos de Norteamérica.

Douglas en 1955 indicó que "en los museos de sitio, el sitio es el elemento más importante en el museo y el edificio debe estar lo más cerca posible al mismo".

Y Lewis en 1959 indica que "un museo de sitio es el que está estrechamente asociado con un lugar que tiene importancia 
científica o histórica. El museo está ubicado en el sitio. Su ámbito de aplicación es la historia y el significado del sitio a los visitantes.

Las colecciones más perecederas se conservan en el sitio. Sus actividades se centran en el sitio. Se ofrecen las instalaciones necesarias para los estudiantes en el sitio, tales como colecciones de estudio, biblioteca y espacios de trabajo".

"Un museo de sitio es un museo diseñado y construido para proteger los bienes naturales y culturales, muebles e inmuebles, in situ, es decir, que se encuentran en el lugar donde fueron creados o descubiertos. Pueden ubicarse en cualquier lugar" (Icom, 1982).

El concepto de museo ha evolucionado a lo largo del tiempo, han pasado a ser desde un lugar exclusivo e impersonal donde se exhibían colecciones diversas, especialmente arte, hasta nuestros días en los que se habla de un museo que no solo conserva, investiga y comunica sino que también educa, entretiene y relaja a sus visitantes (Sola, 1997).

Estas últimas funciones están relacionadas directamente con el público; si el museo no cumple con ellas, entonces deja de cumplir con su misión principal que es, justamente, el encuentro directo con el público (Hernández, 1994).

Una de las principales y más duras críticas al museo es que solo permitía un acercamiento pasivo del público, en actitud contemplativa, promoviendo un hálito de silencio y sacralización de lo exhibido.

Debido a los cambios en la sociedad cada vez va cobrando más importancia la cultura y el deseo de ser parte activa en ella. Por consiguiente, los museos han debido convertirse en lugares abiertos a la interacción del público con la colección, a través de actividades cuyos fines básicos son el acercamiento a la colección y el disfrute del tiempo en el museo.

Uno de los riesgos que se presentan frente a esta realidad es que, por el afán de satisfacer al público o hacer crecer la demanda de visitantes, comience a primar el "espectáculo sobre el valor histórico y artístico del museo" (Alvarez, 2011), desdibujándose, de esta manera, la función comunicadora del mismo.

Los elementos de un museo deben ser considerados base para su análisis y reflexión sobre el rol que cumple el museo en la sociedad, los cuales son:

-El edificio

- Las colecciones

-El personal

-El público

- La conservación

- La restauración

\section{MATERIALES Y MÉTODOS}

El tipo de estudio que se realizó es descriptivo donde se han descrito todas las dimensiones de estudio, se expresó a través de una encuesta a los estudiantes de turismo de tres institutos de educación superior y tres universidades que imparten dicha carrera en su formación profesional, la encuesta aplicada fue validada a través de juicio de expertos.

La Población estuvo comprendida por 1861 estudiantes de la carrera de turismo de universidades (1354) e institutos 
(507) en la ciudad de Lima Metropolitana. La Muestra estuvo conformada por 319 estudiantes de turismo de diversas instituciones educativas.

Las técnicas de recolección de datos fueron las encuestas conformadas por 14 preguntas dirigida a los estudiantes de tu- rismo de las 3 universidades y 3 institutos de la ciudad de Lima y fueron analizadas a través de la herramienta Google forms.

Asimismo, logramos identificar las características de la muestra en función al género y grupo etario, tal como se muestra a continuación:

Tabla 1. Género

\begin{tabular}{ccc}
\hline Género & Cantidad & Porcentaje \\
\hline Masculino & 120 & 37,6 \\
Femenino & 199 & 62,4 \\
Total & 319 & 100,0 \\
\hline
\end{tabular}

Al respecto se detalla que el $37,6 \%$ de los encuestados manifestaron pertenecer al género masculino, los mismos que estuvieron conformados por 120 participantes, mientras que el $62,4 \%$ de las encuestadas manifestaron pertenecer al género femenino, las mismas que estuvieron conformadas por 199 participantes.

Tabla 2. Edad de los estudiantes de turismo en la ciudad de Lima Metropolitana

\begin{tabular}{ccc}
\hline Edad & Cantidad & Porcentaje \\
\hline $0-20$ & 89 & 27,9 \\
$21-40$ & 221 & 69,3 \\
$41-60$ & 9 & 2,8 \\
Más de 60 & 0 & 0,0 \\
Total & 319 & 100,0 \\
\hline
\end{tabular}


Al respecto esta registra que los grupos etarios estuvieron conformados de la siguiente manera:

• El 27,9\%, los mismos que están conformados por 89 participantes oscilan entre los " 0 - 20" años. El 69,3 \%, los mismos que están conformados por 221 participantes oscilan entre los "21 - 40" años. El 2,8 \%, los mismos que están conformados por 9 participantes oscilan entre los "41 - 60" años.

\section{RESULTADOS}

Tabla 3. Régimen de visitas a los museos por parte de los estudiantes de turismo en la ciudad de Lima metropolitana pertenecientes al centro de formación profesional en turismo

\begin{tabular}{ccccc}
\hline Centro de formación & $\begin{array}{c}\text { Total de } \\
\text { encuestas }\end{array}$ & Pachacamac & Puruchuco & Pucllana \\
\hline Universidad privada (A) & 66 & 16 & 18 & 32 \\
Universidad privada (B) & 87 & 43 & 22 & 22 \\
Universidad nacional (A) & 48 & 21 & 15 & 12 \\
Instituto (A) & 19 & 7 & 4 & 8 \\
Instituto (B) & 36 & 12 & 12 & 12 \\
Instituto (C) & 63 & 21 & 22 & 20 \\
TOTAL GENERAL & 319 & $\mathbf{3 7 , 6 2 \%}$ & $\mathbf{2 9 , 1 5 \%}$ & $\mathbf{3 3 , 2 3} \%$ \\
\hline
\end{tabular}

Al respecto esta registra el régimen de visitas a los museos por parte de los encuestados pertenecientes al Centro de Formación Profesional en Turismo el $22 \%$ visito Puruchuco, la Universidad privada (A) el $32 \%$, visito el museo de sitio Huaca Pucllana, la universidad privada (B) el $43 \%$, visitó el museo de sitio Pachacamac, la universidad nacional (A) el $21 \%$, visitó el museo de sitio Pachacamac, el instituto $(A)$ el $8 \%$, visitó el museo de sitio Huaca Pucllana, el Instituto (B) el $12 \%$, visitó el museo de sitio Pachacamac, museo de sitio Puruchuco y el museo de sitio Huaca Pucllana. 
Tabla 4. Mejoras acontecidas con el paso de los años a los museos de sitio Pachacamac, Puruchuco y Huaca Pucllana según la percepción de los estudiantes de turismo en la ciudad de Lima Metropolitana

\begin{tabular}{lccc}
\hline \multicolumn{1}{c}{ Actividades } & M. Pachacamac & M. Puruchuco & M. Pucllana \\
\hline Exhibiciones & 19 & 4 & 9 \\
Salas & 34 & 6 & 12 \\
Edificios & 5 & 6 & 8 \\
Personal & 6 & 2 & 6 \\
Ubicación de áreas comunes & 6 & 6 & 6 \\
Tipos de visitantes & 6 & 9 & 8 \\
Uso de tecnología & 2 & 15 & 5 \\
Promoción de volantes & 4 & 11 & 12 \\
Promoción por internet & 6 & 8 & 11 \\
Promoción por redes & 8 & 2 & 12 \\
Talleres & 2 & 6 & 17 \\
Restaurantes & 0 & 6 & 7 \\
Cafeterías & 0 & 2 & 8 \\
Total encuestas & 100 & 89 & 130 \\
Total porcentaje & $31,3 \%$ & $40,8 \%$ \\
\hline
\end{tabular}

Al respecto esta registra las mejoras acontecidas con el paso de los años a los museos de sitio Pachacamac, Puruchuco y Huaca Pucllana:

- Respecto al museo de sitio Pachacamac. El $31,3 \%$ de los encuestados manifestaron que se han experimentado mejoras respecto a los años anteriores.
- Respecto al museo de sitio Puruchuco. El $27,9 \%$ de los encuestados, manifestaron que se han experimentado mejoras respecto a los años anteriores.

- Respecto al museo de sitio Huaca Pucllana. El 40,8 \% de los encuestados, manifestaron que se han experimentado mejoras respecto a los años anteriores. 
Tabla 5. Evaluación del "Diseño De La Exhibición Permanente" para los Museos de Sitio Pachacamac, Puruchuco Y Huaca Pucllana, por parte de los estudiantes de turismo de la Ciudad de Lima Metropolitana

\begin{tabular}{lcccc}
\hline \multicolumn{1}{c}{ Centro de Formación } & $\begin{array}{c}\text { Total de } \\
\text { Encuestas }\end{array}$ & Pachacamac & Puruchuco Pucllana \\
\hline Universidad privada (A) & 66 & 6 & 6 & 6 \\
Universidad privada (B) & 87 & 7 & 6 & 5 \\
Universidad nacional (A) & 48 & 6 & 6 & 6 \\
Instituto (A) & 19 & 6 & 6 & 6 \\
Instituto (B) & 36 & 6 & 6 & 6 \\
Instituto (C) & 63 & 6 & 6 & 6 \\
TOTAL GENERAL & $\mathbf{3 1 9}$ & $\mathbf{6 , 1 7}$ & $\mathbf{6 , 0 0}$ & $\mathbf{5 , 8 3}$ \\
\hline
\end{tabular}

Respecto a la evaluación del "Diseño de la exhibición permanente" para los museos de sitio Pachacamac, Puruchuco y Huaca Pucllana, por parte de los estudiantes de turismo de la ciudad de Lima metropolitana. El promedio ponderado producto de esta evaluación, resultó de la siguiente manera:

- Museo de Sitio Pachacamac: 6,17 muy satisfecho.

- Museo de Sitio Puruchuco: 6,00 muy satisfecho.

- Museo de Sitio Huaca Pucllana: 5,83 satisfecho.

Respecto a la evaluación de la "limpieza y mantención de la exhibición", para los museos en estudio los estudiantes indicaron que la evaluación en el museo de sitio Pachacamac: 6,49 muy satisfecho; museo de sitio Puruchuco: 6,57 muy satisfecho; museo de sitio Huaca Pucllana: 6,48 muy satisfecho.

Respecto a la evaluación de la "recepción, acogida y trato recibido de parte de los funcionarios del museo", para los museos en estudio, los estudiantes indicaron museo de sitio Pachacamac: 4,89 medianamente satisfecho; museo de sitio Puruchuco: 4,86 medianamente satisfecho; museo de sitio Huaca Pucllana: 4,89 medianamente satisfecho.

Respecto al museo de sitio de Pachacamac, el 33,86\% de los estudiantes se encuentran "medianamente satisfechos".

Respecto al museo de sitio de Puruchuco, el $40,13 \%$ de los estudiantes se encuentran "medianamente satisfechos". Respecto al museo de sitio Huaca Pucllana, el 26,02 \% de los estudiantes se encuentran "medianamente satisfechos". 


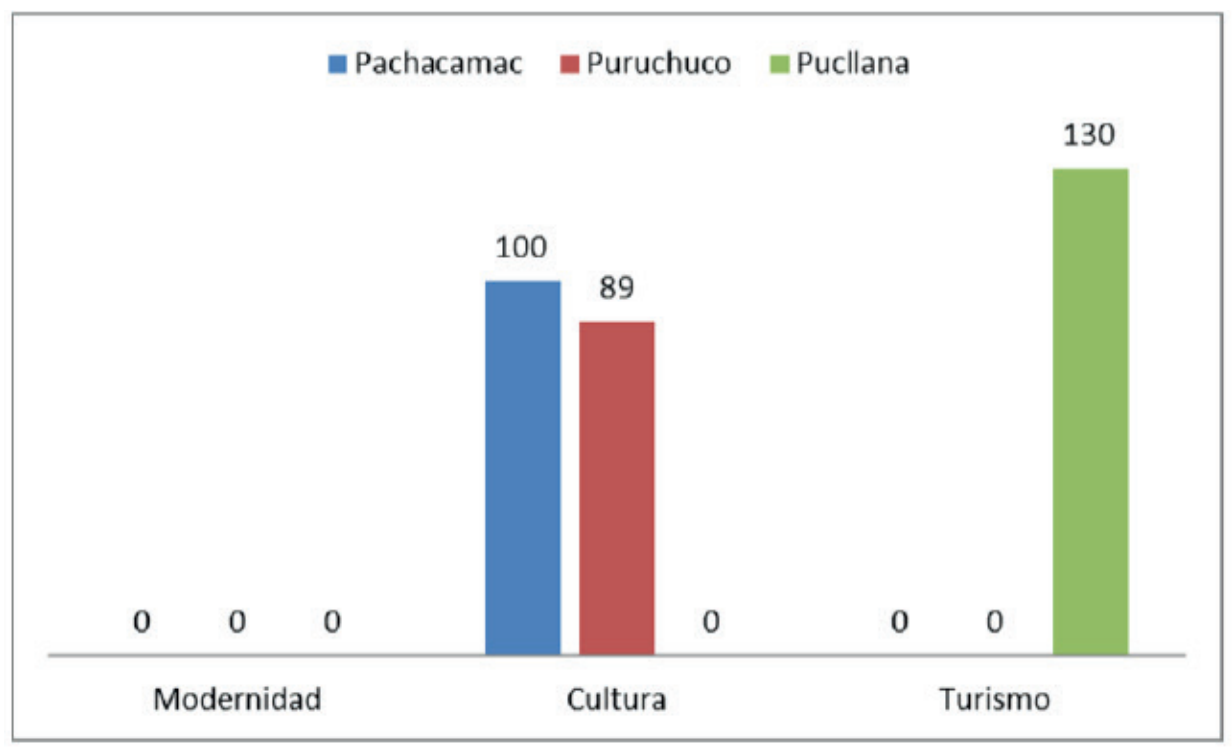

Figura 1. La Palabra más adecuada para relacionar a los museos de sitio Pachacamac, Puruchuco y Huaca Pucllana

El $62,70 \%$ de los estudiantes manifestaron que la palabra más adecuada para relacionar a los museos de sitio Pachacamac, Puruchuco y Huaca Pucllana es "cultura".

El 37,30 \% manifestaron que la palabra más adecuada para relacionar a los museos de sitio Pachacamac, Puruchuco y Huaca Pucllana es "turismo".

\section{DISCUSIÓN}

Consideramos conveniente abordar sobre la importancia de la museología para la educación, la identidad nacional y la formación de generaciones que en un corto tiempo serán los que lleven los destinos de la cultura y el turismo en nuestro país.

El museo ha estado ligado a la educación desde sus orígenes y eso parece estar muy claro para los estudiantes de la carrera de turismo y en ello se ha venido trabajando a tal punto que los museos de sitio Pachacamac, Puruchuco y Huaca Pucllana así lo han demostrado con esta investigación.
Sin embargo, no son pocas las investigaciones que señalan la necesidad de incrementar las cifras de visitantes hacia los museos con fines educativos, fue lo que llevó a algunos de los museos investigados a mejorar su gestión e innovar en el servicio y el buen trato, lo que requiere de un incremento presupuestal y los museos públicos no han sido ajenos a los presupuestos, las estadísticas, los mismos que a su vez han determinado en el éxito o el fracaso de cualquier actividad dentro de los museos.

Es de interés conocer la percepción que pueden tener los estudiantes de la carrera de turismo y los estudiantes en general, más aún a la administración del museo para evaluar las metas institucionales de los museos.

\section{CONCLUSIONES}

Es una oportunidad para identificar si los estudiantes de turismo realizan visitas a museos.

Es oportuno para revisar las mallas curriculares e identificar las actividades de campo 
como visitas a los museos de la ciudad como parte de la formación profesional.

Es aplicable para conocer cuáles son los puntos fuertes y débiles de las condiciones de los museos y de las cosas que los motivan a ser visitados.

Los museos de sitio Pachacamac, y Huaca Pucllana han modificado sus productos con el paso de los años, principalmente en infraestructura y estrategias de muestra de colección.

Los estudiantes de turismo de la universidad privada $(A)$, universidad privada $(B)$, universidad nacional $(A)$ y de los instituto $(A)$, instituto $(B)$, instituto $(C)$ no conocen el museo de Puruchuco en su mayoría.

\section{REFERENCIAS BIBLIOGRÁFICAS}

Alvarez, C. (2011). Museos pedagógicos: ¿Museos didácticos? España: Gazeta de Antropología.

Amoros, R. (2017). El Museo de sitio Arturo Jiménez Borja - Puruchuco, diagnóstico y lineamientos para mejorar su propuesta museográfica.

Chirinos, V. (2013). Propuesta museográfica para el museo de sitio Huaca Pucllana: Reflexiones sobre el vínculo entre el pasado y el presente de Lima Metropolitana.

Gobierno Municipal- Pachacamac. (sin fecha). Obtenido de Gestión de la Municipalidad-Pachacamac: http://www.munipachacamac.gob.pe/limites.html.

Hernández, F. (1994). El Museo como espacio de comunicación. Asturias: Ediciones Trea.
Icom. (1982). Revista de Patrimonio. Obtenido de http://www.revistadepatrimonio.es/ revistas/numero1/institucionespatrimonio/ estudios/articulo3.php.

Jimenez, A. (1988). Puruchuco. Lima: Serie Perulibros.

Ministerio de Cultura. (2010). www.mcultura.gob.pe. Obtenido de http://www.mcultura. gob.pe/documentosweb/c33f21060d2e664f5af5c231d046c21a/Gaceta_39_OK.pdf.

Miraflores, M. D. (2018). http://huacapucllanamiraflores.pe/historia-2/.

Sola, T. (1997). La formación inicial y su incidencia en la educación especial. Madrid: Pirámide. 